\title{
To investigate the correlation of proton leak and current produced from animal cells by microbial fuel cells
}

\author{
Karen Poon ${ }^{1}$, Tse Chiu Chung ${ }^{1}$, Chang $\mathrm{Xu}^{1,2}$, Ruihua Wang ${ }^{3, *}$ \\ ${ }^{1}$ Program of Food Science and Technology, Division of Science and Technology, BNU-HKBU United International College, 28 Jinfeng \\ Road, Tangjiawan, Zhuhai, Guangdong, China \\ ${ }^{2}$ Department of Chemistry, Hong Kong Baptist University, 224 Waterloo Road, Kowloon Tong, Hong Kong SAR, P.R. China \\ ${ }^{3}$ Department of Gastroenterology, Shanghai Jiao Tong University affiliated Sixth People's Hospital South Campus, 6600 Nanfeng Road, \\ Fengxian District, Shanghai, China
}

\section{Email address:}

karenpoon@uic.edu.hk (K. Poon), f031300064@mail.uic.edu.hk (T. C. Chung),janexu@uic.edu.hk (Chang Xu), ruihuawang@sina.cn (Ruihua Wang)

\section{To cite this article:}

Karen Poon, Tse Chiu Chung, Chang Xu, Ruihua Wang. To Investigate the Correlation of Proton Leak and Current Produced from Animal Cells by Microbial Fuel Cells. American Journal of Life Sciences. Vol. 2, No. 3, 2014, pp. 176-181. doi: 10.11648/j.ajls.20140203.17

\begin{abstract}
Proton leak has been implicated in various chronic diseases like diabetes and cancer. In this study, current from intact cells, including mice liver cells, pig blood cells and human breast cancer cell MCF-7 were measured by microbial fuel cells (MFC). Positive current change in normal liver cells were induced by either 2,4-dinitrophenol (DNP) or Piceatonnol. The effect of DNP in enhancing the proton conductivity would increase the degree of positive current change, while Piceatonnol in improving the mitochondria membrane potential would support the sustainability of the positive current change with time. Piceatonnol was found to be more effective in inducing positive current change in cancer cells than in liver cells. The higher effectiveness of Piceatonnol to cancer cells would be explained by the high proton leak condition of the cells, and so increased the current production. Little positive current change could be induced in red blood cell by either DNP or Piceatonnol. Results supported the hypothesis of the high mitochondria membrane potential to support the positive current change in cells with time, while the proton conductivity determined the degree of positive current change. The condition of proton leak of cells seemed to be the limiting factor for the positive current change in cells.
\end{abstract}

Keywords: Proton Leak, Electron Leak, Microbial Fuel Cells, 2,4-Dinitrophenol, Piceatonnol

\section{Introduction}

In the respiratory metabolism, oxidation of substrates produced electron and proton that would either be used directly by ADP to form ATP or convert the cofactor NAD to NADH in cytoplasm. The oxidative form NADH would be recycled back to $\mathrm{NAD}^{+}$with the production of electrons and protons via the electron transport chain in the mitochondria. The chemical process of oxidative phosphorylation in mitochondria would pass electrons through three complexes containing two electron carriers (ubiquinol and cytochrome c) (1) in the electron transport chain, while the protons would be pumped outside the mitochondrial matrix to the inner mitochondrial membrane. The building up of high mitochondrial membrane potential allowed the proton flow (2) from inner membrane via the complex IV back to the matrix have enough energy to convert ADP to ATP, and finally accepted by the molecular oxygen to water (3). Majority of ATP, about $90 \%$ were generated in the final step of electron transport chain (3).

However, not all the electrons would pass the electron transport chain, some of them might escape at earlier steps. Studies revealed that higher metabolic rate would increase the chance of electron leaking (4) and fasting would lower the level (5). The sluggish movement of electron transfer would also increase the chance of electron leaking away from its normal pathways and escaped to other part of the body. Although we did not know where these electrons are all end up to, some of them were used to generate heat energy, and $50 \%$ of the escaped electron would form superoxide by single electron reduction of molecular oxygen, which was a major reactive oxygen species (ROS) in cells (6). The loss of the electrons would account for the energy loss and basal metabolism. The accumulation of 
ROS cells accounted for the major reason of apoptosis (7).

Apart from electron leak, proton leak was also observed during the process of oxidative phosphorylation that was found incompletely coupled in particular in those diseased cells including cancers and diabetes. Electrons and protons could leak across the inner membrane and relieve proton-motive force independently of ATP synthase (8). The proton leak was also found to be induced by ROS production of cell (9). One of the benefits of proton leak was to behave as an antioxidant and reduce ROS production (10). The up-regulation of uncoupling protein 2 (UPC2) in cancer (7) promoting the proton leak explained the enhancement of cell protection against chemotherapeutic agents (11). Increased proton leak and decreased ROS were observed in cancer cells (7). Cellular ROS levels can be effectively controlled by the rate of proton leak (12). Some of the protons might leak out and flow back to the mitochondrial matrix, the "mild uncoupling" (proton leak) occurred in the animal's body that slightly decreased membrane potential thereby preventing fast production of reactive oxygen species in mitochondria. Subsequently, it would decrease the damage and aging of the cells (13).

Uncoupling proton decreases mitochondrial ROS release through many mechanisms (4). First, the oxygen consumption would increase with higher respiratory rates. It might possibly result in lower oxygen tensions in the mitochondrial microenvironment. This decreases the probability of one electron reduction of oxygen at the electron transport chain, generating the superoxide radical anion, the primary ROS produced by mitochondria (14). The second effect of enhanced respiratory rates on ROS release is the maintenance of electron transport intermediates, especially for the complex I and III, which are the more oxidized state that cannot donate electron to oxygen, producing superoxide radical (15). Lower mitochondrial inner membrane potentials also decrease the occurrence of reverse electron transfer from complex II to complex I, a major source of ROS in many tissues (16). Reverse electron transfer is thermodynamically feasible at high inner membrane potentials, which compensate for the differences in redox potential between complexes I and II. Finally, increased respiratory rates decrease ROS release by pyruvate and $\alpha$-ketoglutarate dehydrogenases in mitochondria, due to increments in the availability of $\mathrm{NAD}^{+}$(17-19). In some cancers, UCP2 is highly abundant and may advance metabolic reprogramming, further disrupt tumor suppression, and promote chemo-resistance (20). Therefore, a simple, convenient and cost-saving electron and proton leak measurement is very important to advance the research on the therapeutic development.

In the present study, microbial fuel cells (MFC) was used to measure the current generated from normal mice liver cells, pig blood cells and human breast cancer cells MCF-7. Live animal cells were suspended in the anode of MFC, while Potassium Hexacyanoferrate (III) was placed in the cathode. The electrons leaking from the cells would pass to the anode, which ended up flowing to the external circuit, while protons leaking from cells would cross the inter-chamber membrane to the cathode to complete the circuit, resulting in current production. In order to simplify the interpretation of data, no mediator was used to enhance the leakage of electron from cells in the experiment. The change of current production by the cells would directly reflect to the action of the added chemicals.

As the ATP consumption of liver cell was very high, a higher electric current could be detected by MFC, which would be useful when comparing data and observed changes amongst different cells. Apart from liver cells were chosen for the experiment, MCF-7 breast cancer cells and pig blood cells were also used. Most of the cancer cells were found to have high proton leaking, which may be due to the unusual up-regulation of UCP2 in mitochondria during the advance stage of cancer development (7). Most of the protons were found to be leaked via the UCP2 protein, which could be an advance metabolic reprogramming in cancer cells to disrupt tumor suppression, and promote chemo-resistance (20). Blood cells were the kind of cells without mitochondria. No electron transport chain and high mitochondrial membrane potential could be found in blood cells. The use of blood cells in the experiment could be used as a control. Protonophore 2,4-dinitrophenol (DNP) inducing proton leak by increasing proton conductivity of cells and Piceatannol increasing mitochondria membrane potential by inhibiting the ATP synthase to ATP oxidative phosphorylation would be used in the study. The study of current generation from animal cells would help us to understand the correlation between current generation and the status of proton leak.

DNP also exhibited favorable effects in some pathological laboratory models related to oxidative stress and increase lifespan of mice (15). Low doses of the DNP promoted enhanced tissue respiratory rates, improved serological glucose, triglyceride and insulin levels, decrease of ROS levels and tissue DNA and protein oxidation, as well as reduced body weight (15). Piceatannol is a stilbene phytochemical from the seeds of Euphorbia lagascae, which have been used widely in folk medicine for treating tumors, cancers, and warts (21). Piceatannol inhibits the F-type ATPase by targeting the F1 sector, which is located to the inner membrane of mitochondria and plasma membrane of normal endothelial cells and several cancer cell lines (22).

\section{Materials and Methods}

\subsection{Materials and Reagents}

All chemicals for experiment were in analytical grade. The essential reagents including ethanol absolute was supplied by Tianjin Damao Chemical Reagent Factory (Tianjin, China). DMEM medium was purchased from HyClone Laboratory Inc. (Beijing, China). Fetal bovine serum (BSA) was obtained from Tianjin Haoyang 
Biological Technology Co., Ltd (Tianjin, China). Trypsin-EDTA was purchased from Life Technologies Corporation (Shanghai, China). The ATP inhibitors, Piceatannol was purchased from Shanghai Yaji Biotech Co. As a protonophore, 2,4-dinitrophennol (DNP) was purchased from Dongfang Chemicals Factory in China. Stock solutions of these standards were made as 5000ppm in ethanol and stored at $-10^{\circ} \mathrm{C}$ before usage. Water was purified by Water purifying system (EPED, China).

\subsubsection{Perfusion Buffer Concentrate (PBC)}

Dissolve $\mathrm{NaCl}(103.75 \mathrm{~g}), \mathrm{KCL}(6.25 \mathrm{~g})$, Hepes (28.70g) in $\mathrm{H}_{2} 0$, added $1 \mathrm{M} \mathrm{NaOH}(75 \mathrm{ml})$ when all dissolved. Add $\mathrm{H}_{2} 0$ to a constant volume $500 \mathrm{ml}$.

\subsubsection{Perfusion Buffer}

Use $40 \mathrm{ml}$ PBC to constant volume to 1 litre by milli-Q water. Then autoclaved the buffer at $120{ }^{\circ} \mathrm{C}$ for 20 minutes.

\subsubsection{Preservation Buffer}

Add $2.5 \mathrm{~g}$ of BSA to $250 \mathrm{ml}$ of perfusion buffer. Need $+4{ }^{\circ} \mathrm{C}$ or on ice store.

\subsubsection{Dissociation Buffer}

Add $0.5 \mathrm{ml}$ of $476 \mathrm{mM} \mathrm{CaCl}_{2}$ to $49.5 \mathrm{ml}$ of perfusion buffer to obtain a final concentration of $4.76 \mathrm{mM} \mathrm{CaCl}_{2}$. Add $3 \mathrm{mg}$ of Collagenase (Biosharp) just before perfusion start.

\subsection{Mice Liver Cell Preparation (23)}

After the mouse was anesthetized with ethyl ether, the fainted mouse was opened to expose the peritoneal cavity to locate the portal vein. Perfusion buffer $(30 \mathrm{~mL})$ was first perfused into the portal vein via an intravenous cannula; the hepatic vein was then cut. After the blood from the liver was cleaned, dissociation buffer $(50 \mathrm{~mL})$ was perfused to the liver via the portal vein.

The liver was removed from the body to an aseptic petri dish with preservation buffer. Gall bladder was carefully removed and the liver cells were then released into the preservation buffer. The liver cell solution was centrifuged four times with different condition. The first centrifugation of the liver cell solution was in the condition $54 \mathrm{x}$ g for $2 \mathrm{~min}$ at $4{ }^{\circ} \mathrm{C}$. The supernatant from the first centrifugation was collected and then was continued to centrifuge in another two minutes in the same condition $\left(54 \mathrm{x}\right.$ g for 2 min at $\left.4{ }^{\circ} \mathrm{C}\right)$. The second supernatant was collected then was centrifuged third time with $1350 \mathrm{x}$ g for $10 \mathrm{~min}$ at $4{ }^{\circ} \mathrm{C}$.

The precipitate was collected and $10 \mathrm{~mL}$ preservation buffer, 25\% Percoll and 50\% Percoll were added into centrifuge tube then centrifuged under $1350 \mathrm{x} g$ for $30 \mathrm{~min}$ at $4{ }^{\circ} \mathrm{C}$. The non-parenchymal cells from the interface, between the two density cushions of $25 \%$ with $50 \%$ Percoll, was collected with a $10 \mathrm{ml}$ pipette. Non-parenchymal cells were re-suspended in $75 \mathrm{ml}$ preservation buffer to make up total $100 \mathrm{ml}$ solution in a conical flask. Transfer $25 \mathrm{ml}$ of the non-parenchymal cells solution to another three conical flasks that contained $75 \mathrm{ml}$ preservation buffer respectively to make 4 equal diluted portions for the test of microbial fuel cell.

\section{3. $\mathrm{MCF}-7$ cell Preparation}

MFC-7 (24), breast cancer cell, was cultured in DMEM with $10 \%$ FBS and $1 \%$ penicillin medium in the carbon-incubator at $37^{\circ} \mathrm{C}$ for two days to allow the cell growth. After the cells were grown to cover the surface of the plate, it was ready to experimental use. The medium of cells were removed and rinsed with $2 \mathrm{ml}$ PBS, before Trypsin was added for re-suspending cells. The harvested cell was added to PBS and frozen at $-80^{\circ} \mathrm{C}$ before use.

\subsection{Pig Blood Cells}

Fresh pig blood cells were purchased from the local farm. Blood cells were washed with saline two times before used.

\subsection{Microbial Fuel Cell (MFC) Test}

Each set of MFCs apparatus consisted of a cathode and an anode chamber. Potassium hexacyanoferrate (III) $(100 \mathrm{ml})$ was placed in the cathode, while the cells were suspended in preservation buffer in the anode chamber. Nitrogen gas was used to remove oxygen in the anode, because the presence of oxygen in anode would decrease the electricity generation (25). Concentrations used for different cells were adjusted until they would give a similar initial voltage value in MFC. $50 \mathrm{mM}$ potassium hexacyanoferrate (III) was used as the terminal electron acceptor in cathode of the system. Proton exchange membrane was used to separate the two chamber anode and cathode. The polymer membrane was permeable to protons when it was saturated with water, but it did not conduct electrons. Proton was allowed to exchange in the system, the circuit was completed. The voltage $\mathrm{E}_{\mathrm{MFC}}$ of the circuit was monitored by the Digital Multi-meter (Tektronix DM4020).

The voltage $E_{M F C}$ measured in MFC was the electric potential between the two electrodes, that is the function of the external resistance $\left(\mathrm{R}_{\text {ext }}\right)$ and the current $\mathrm{I}$. The relationship was represented as follows:

$$
\mathrm{E}_{\mathrm{MFC}}=\mathrm{I} \mathrm{R}_{\mathrm{ext}}
$$

The external resistance $\mathrm{R}_{\mathrm{ext}}$ referred to the resistance loaded on the external circuit, while the current I was the current flowing to the external circuit. As the current produced from MFC in the external circuit was small, the current was usually estimated by calculation from the measured voltage.

For $\mathrm{R}_{\mathrm{ext}}=5000 \mathrm{ohm}$

$\mathrm{I}=\mathrm{E}_{\mathrm{MFC}} / 5000 \mathrm{ohm}$

As the internal resistance of MFC would be influenced by the condition of the MFC, it in terms would affect the overall electric power generation (25). Under the same condition of MFC, the internal resistance was assumed to remain the same. Voltage or current change would reflect the action of the chemicals added to the system. System loaded with external resistance of $5000 \mathrm{ohm}$ was used for the calculation. The 20 minute current value before the experiment was used as the baseline control, while the 
current change was calculated by subtracting the 20 minute current value after the addition of chemical with the 20 minute control value.

\section{Results}

\subsection{Figure (1 \& 2)}

DNP was found to induce positive current change in MFC placed with normal liver cells in a dose-dependent manner (Fig. 1). Low dose of DNP at $10 \mathrm{ppm}$ or $15 \mathrm{ppm}$ did not induce high positive current change, but at the high dose of $20 \mathrm{ppm}$. Higher positive current change was observed at $20 \mathrm{ppm}$, which was then gradually declining over the next 20 minute. (Fig. 1) Low dose at 10 ppm was found to decrease the current production and yield negative current change.

Piceatonnol also induced positive current change in normal liver cells (Fig. 2). Low dose at 10ppm produced negative current change, while higher dose at $15 \mathrm{ppm}$ or $20 \mathrm{ppm}$ produced positive current change. Higher dose at $20 \mathrm{ppm}$ induced more positive current change than that of the low dose of $15 \mathrm{ppm}$ (Fig. 2).

Both DNP and Piceatonnol would induce positive current change in normal liver cells in a dose-dependent manner (Fig. $1 \& 2$ ). The positive current change was found to be higher in DNP than in Piceatonnol treated cells (Fig. 1 \& 2). The positive current change in DNP was found in a declining mode with time (Fig. 1), while that in Piceatonnol was in an increasing mode with time.

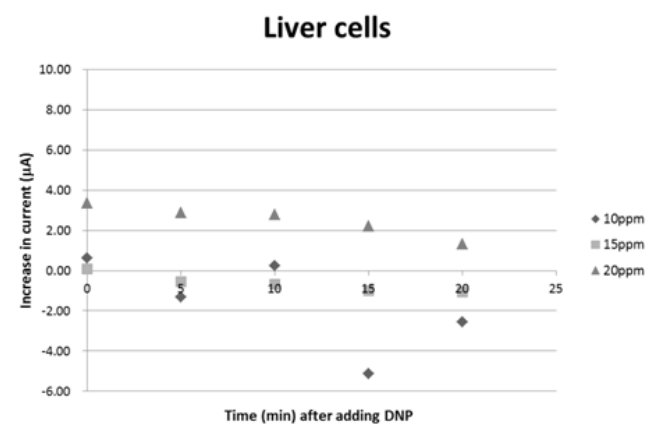

Figure 1. Increase in current produced from liver cells with time after DNP (10ppm, 15ppm, 20ppm) was added.

\section{Liver cells}

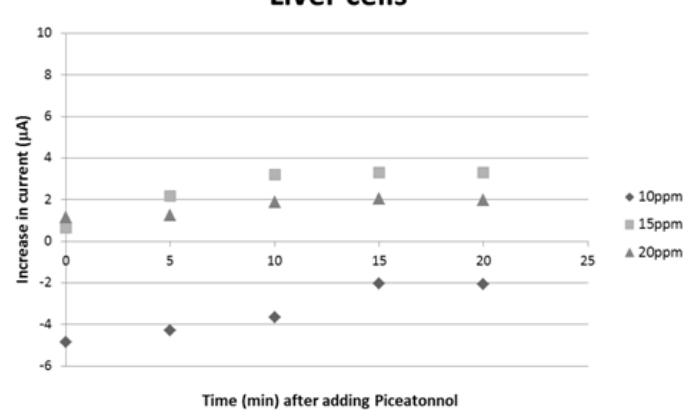

Figure 2. Increase in current produced from liver cells with time after Piceatonnol (10ppm, 15ppm, 20ppm) was added.

\subsection{Figure $(3 \& 4)$}

The positive current changes induced by DNP in the human breast cancer cells MCF-7 was similar to that in liver cells (Fig. 1,3), while the positive current changes induced by Piceatonnol were found to be much higher in cancer cells (Fig. 2,4) than in liver cells. Similar to the liver cells, DNP produced positive current change in a declining mode with time (Fig. 3), while Piceatonnol was in an increasing mode (Fig. 4). The effectiveness of Piceatonnol to induce positive current change in $\mathrm{MCF}-7$ cancer cells was found ro be higher than that of DNP (Fig. $3 \& 4$ ).

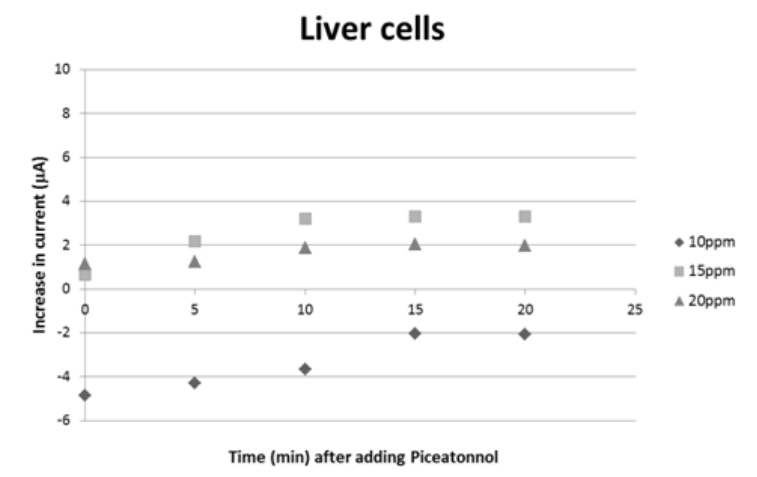

Figure 3. Increase in current produced from MCF-7 cancer cells with time after DNP (20ppm) was added.

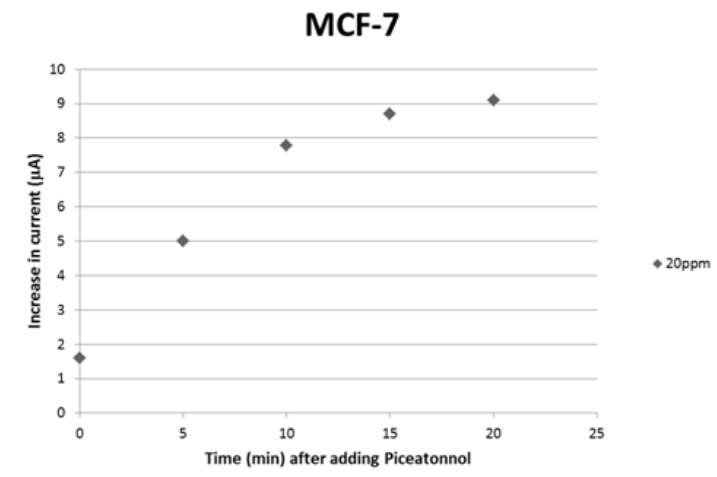

Figure 4. Increase in current produced from MCF-7 cancer cells with time after Piceatonnol (20ppm) was added.

\subsection{Figure $(5 \& 6)$}

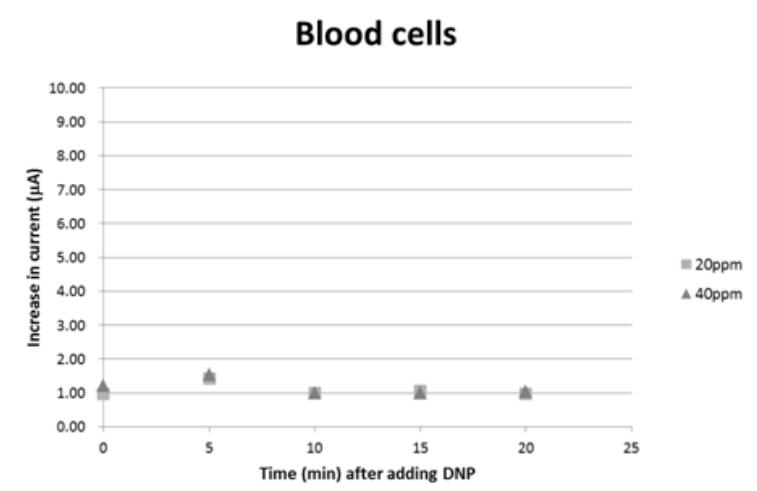

Figure 5. Increase in current produced from blood cells with time after DNP (20ppm, 40ppm) was added. 


\section{Blood cells}

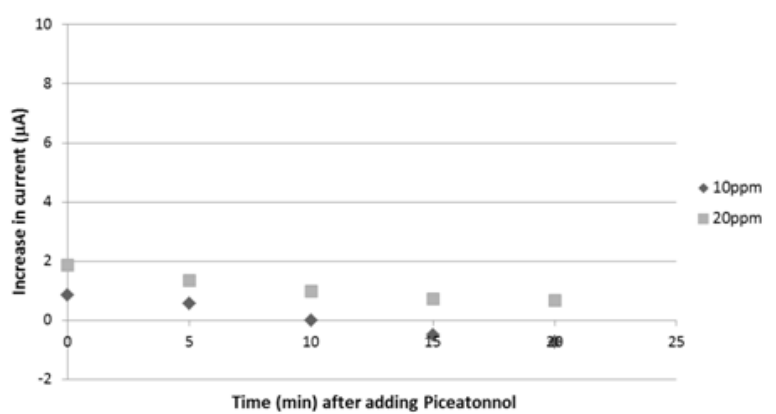

Figure 6. Increase in current produced from blood cells with time after Piceatonnol (10ppm, 20ppm) was added.

Blood cells were found to have little response to either DNP or Piceatonnol. Little positive current changes could be induced by two of the chemicals (Fig. 5 \& 6), which were much lower than that found in normal liver cells or cancer cells. (Fig. 1-6)

\section{Discussion}

MFC was a system that would utilize the bacterial metabolism to generate electricity from different substrates. Similar to other bacteria, animal cells were found to generate electric current during the cellular metabolism (26). Electron and proton were produced during glycolysis and Kreb's cycle would either directly be utilized to form ATP in cytoplasm, or form NADH from $\mathrm{NAD}^{+}$. In the mitochondria, NADH will convert back $\mathrm{NAD}^{+}$to generate electrons and proton in the electron transport chain. Protons would be pumped to the inner membrane of mitochondria and increase the mitochondria membrane potential (2). The high proton-motive force would drive protons flow from the inner membrane back to the mitochondrial matrix via the ATP synthase and convert ADP to ATP (2). However, not all the proton would flow back to the mitochondrial matrix via the ATP synthase, some of them would escape and leak out from the inner membrane. The leaking of the protons led to energy loss. Some of the energy loss from proton leak was released as heat to bodies, which account for $20-30 \%$ of heat generation of basal metabolism (27). The situation was found to be more severe in chronic disease cells, e.g. diabetes and cancer cells (28).

Protonophore DNP would enhance the proton leaking by increasing the proton conductivity across the inner membrane of the mitochondria. The leaking of proton was found to correlate with the decreased level of ROS, tissue DNA and protein oxidation (15). The leaked proton would behave as an antioxidant to react with the surrounding free radicals, which in terms reduced the amount of reactive oxygen species in cells. It was the situation observed in the high proton leaked cancer cells, in which the leaked proton was used to decrease ROS production (7). Although there was no free radicals in MFC to react with the leaked proton, the residual amount of oxygen existed in the anode chamber would react with the proton and so decrease the electricity generation (25). The above reason might explain the reason why not so much of leaked proton could reach the cathode at low dose of DNP. However, when increasing the dose of DNP, more protons would be leaked out from cells at a time, which would bring along the electron out from the cells for charge balance, and so complete the circuit. Current could then be produced by the cells. The current measured from cells were found to be dose-dependent on DNP.

Under normal condition, high proton leak was not observed in normal cells, except in diseased cells, e.g. diabetes or cancer cells. In cancer cells, UCP2 was up-regulated and proton leak was found to go through the UCP2 channel. Therefore, when Piceatonnol increased the mitochondrial membrane potential by inhibiting ATP synthase, increase in positive current change was observed in cells. The effect was found to be much higher in cancer cells than in normal liver cells, as the proton conductivity was much higher in cancer cells than in normal liver cells. The increase in current production by cells was found to be limited by the flow of proton.

Current production also depended on the continuous supply of electron and proton from cells. ATP synthase inhibitor Piceatonnol would block the re-entry of proton from inner mitochondrial membrane to the matrix via the ATP synthase to form ATP from ADP. The effect of Piceatonnol would allow the proton accumulate in the inner membrane and create a very high mitochondrial membrane potential. The high electro-gradient would increase the tension of proton leaking out from the cell. Results indicated that increase in mitochondria membrane potential by increasing the dose of Piceatonnol would increase the current production with time, while the increase in proton conductivity by DNP would increase the initial current magnitude. The stability of the current magnitude was influenced by the continuous maintenance of high mitochondria membrane potential.

For blood cells without mitochondria did not have mitochondria membrane potential was found to produce little current. The action of DNP to increase proton conductivity on blood cells could only produce small positive current change. Piceatonnol could not improve the mitochondria membrane potential in the blood cells that did not contain mitochondria, and so little positive current change would be induced.

\section{Conclusion}

In this study, we have demonstrated the use of MFC to measure the current change from animal cells and correlate the result with the condition of proton leak. Results indicated that DNP could increase the proton conductivity would induce positive current change from cells and the magnitude of the current change was influenced by the degree of proton conductivity. Piceatonnol could increase the mitochondria membrane potential would determine the sustainability of the positive current change with time. 
Using MFC to measure the current change in cells could help us to correlate the condition of the proton leak in cells and would enhance the advance in research and drug development.

\section{Acknowledgements}

Authors would like to thank UIC College Research Grant R201406 for supporting this project.

\section{References}

[1] Hames, D. \& Hooper, N., "Electron Transport and Oxidative Phosphorylation" In: Biochemistry. s.l.:Garland Science, pp. 398-412, 2011.

[2] Li, X. , Zhao, J. "An Approach to the Nature of Qi in TCM-Qi and Bioenergy." In: H. Kuang, ed. Recent Advances in Theories and Practice of Chinese Medicine. S.l: InTech, pp 79-106, 2012.

[3] Byrd-Bredbenner, C., Moe, G., Beshgetoor, D. \& Berning, J. eds. "Energy Metabolism" In: Wardlaw's Perspectives in Nutrition. The Eighth Edition ed. s.l.:McGraw-Hill, pp. 281-291, 2009.

[4] Skulachev VP "Uncoupling: new approaches to an old problem of bioenergetics." Biochim. Biophys. Acta 1363, 100-124, 1998.

[5] Trzcionka M; Withers KW; Klingenspor M; Jastroch M, "The effects of fasting and cold exposure on metabolic rate and mitochondrial proton leak in liver and skeletal muscle ofan amphibian, the cane toad Bufo arinus." J Exp Biol. 211: pp. 1911-8, 2008.

[6] Brand MD, Affourtit C, Esteves TC, Green K, Lambert AJ, Miwa S, Pakay JL, Parker N "Mitochondrial superoxide production, biological effects, and activation of uncoupling proteins" Free Radic Biol Med 37: 755-767, 2004.

[7] Baffy G "Uncoupling protein-2 and cancer. Mitochondrion" 10: 243-252, 2010.

[8] Ajit S. Divakaruni and Martin D. Brand. "The Regulation and Physiology of Mitochondrial Proton Leak." Physiology 26: 192-205, 2011.

[9] Liu S; Jiao X; Wang X; Zhang L "Interaction of electron leak and proton leak in respiratory chain of mitochondria--proton leak induced by superoxide from an electron leak pathway of univalent reduction of oxygen." Sci China C Life Sci. Vol. 39 (2), pp. 168-78, 1996.

[10] Bevilacqua L., Ramsey J.J., Hagopian K., Weindruch R. , Harper M. "Long-term caloric restriction increases UCP3 content but decreases proton leak and reactive oxygen species production in rat skeletal muscle mitochondria" American Journal of Physiology - Endocrinology and Metabolism. 289. E429-E438, 2005.

[11] Derdak Z, Mark NM, Beldi G, Robson SC, Wands JR, Baffy $\mathrm{G}$ "The mitochondrial uncoupling protein-2 promotes chemoresistance in cancer cells." Cancer Res 68: 2813-2819, 2008.
[12] Brand MD "The proton leak across the mitochondrial inner membrane" Biochim Biophys Acta 1018: 128-133, 1990.

[13] Antonenko YN, Khailova LS, Knorre DA, Markova OV, Rokitskaya TI, "Penetrating Cations Enhance Uncoupling Activity of Anionic. Protonophores in Mitochondria." PLoS ONE 8(4): e61902. 2013 doi:10.1371/journal.pone.0061902.

[14] Balaban RS, Nemoto S, Finkel T "Mitochondria, oxidants, and aging." Cell 120, 483-495, 2005.

[15] Caldeira da Silva CC, Cerqueira FM, Barbosa LF, Medeiros $\mathrm{MH}$, Kowaltowski AJ "Mild mitochondrial uncoupling in mice affects energy metabolism, redox balance and longevity.” Aging Cell 7: 552-560, 2008.

[16] Turrens JF "Mitochondrial formation of reactive oxygen species.” J. Physiol. 552, 335-344, 2003.

[17] Starkov AA, Fiskum G, Chinopoulos C, Lorenzo BJ, Browne SE, Patel MS, Beal MF "Mitochondrial alpha-ketoglutarate dehydrogenase complex generates reactive oxygen species.” J. Neurosci. 24, 7779-7788, 2004.

[18] Tretter L, Adam-Vizi V "Generation of reactive oxygen species in the reaction catalyzed by $\alpha$-ketoglutarate dehydrogenase.” J. Neurosci. 24, 7771-7778, 2004.

[19] Tahara EB, Barros MH, Oliveira GA, Netto LES, Kowaltowski AJ "Dihydrolipoyl dehydrogenase as a source of reactive oxygen species inhibited by caloric restriction and involved in Saccharomyces cerevisiae aging. "FASEB J. 21, 274-283, 2007.

[20] Baffy G, Z Derdak and SC Robson "Mitochondrial recoupling: a novel therapeutic strategy for cancer?" British Journal of Cancer 105, 469-474, 2011.

[21] Hartwell, J. L. Lloydia 32, 153-205, 1969.

[22] Zheng Jianbiao and Victor D. Ramirez. "Piceatannol, a Stilbene Phytochemical, Inhibits Mitochondrial F0F1-ATPase Activity by Targeting the F1 Complex," Biochemical and Biophysical Research Communications 261, 499-503, 1999.

[23] Smedsrød B "Protocol for Preparation of Mouse Liver Kupffer Cells and Liver Sinusoidal Endothelial cells “ 2012 munin.uit.no/bitstream/handle/10037/4575/article.pdf.

[24] Soule, HD; Vazquez J, Long A, Albert S, Brennan M. “A human cell line from a pleural effusion derived from a breast carcinoma." Journal of the National Cancer Institute 51 (5): 1409-1416, 1973.

[25] Logan B. "Voltage generation". In Microbial Fuel cell, John Wiley \& Son, Inc. New Jersey pp. 29-43, 2008.

[26] Li Z., Zhang X., and Lecheng L.L. "Electricity production during the treatment of real electroplating wastewater containing Cr6+ using microbial fuel cell." Process Biochem. 43: 1352-1358, 2008.

[27] Song, Z., Wang, D. "Proton Leak and its Role in Basal Metabolism.” Prog. Biochem. Biophys., 28, pp. 474-477, 2001.

[28] Weindruch R and Sohal RS. "Caloric intake and aging." N Engl J Med 337: 986-994, 1997. 